Nach sinngemäßer Bezitferung ergibt die so entstandene Hauptafel den Inhalt von Gleichung (3), d. h. man kann bei (durch Festlegung von $\eta$ und $\Delta D$ gegebener Konstanten durch Drehung eines angelegten Lineals um den so bestimmten Punkt der F. Leiter auf den $r$ und $v$, L-Leitern die einander zugeordneten Werte ablesen. Vorteilhaft stattet man hierbei die L-Leiter noch mit einer übergelagerten Teilung aus, aus der die Lebensdauern auch nach Minuten, Stunden, Tagen, Monaten, Jahren und Jahrhunderten abzulesen sind. Ebenso kann man an der $r$-Leiter die TeilchengröBe $\mathbf{d}$ direkt ablesbar machen.

In gleich einfacher Weise entsteht die Nebentafel zur F-Leiter für die rechte Seite der Gleichungen (5). Wählen wir, was vorteilhaft ist, für die $\triangle D$-Leiter den gleichen Maßstab, wie er sich für die F-Leiter ergab, dann fällt (s. o.) die $\eta$-Leiter wieder in die Mitte und hat wieder den halben Maßstab. Entsprechend dem geringeren Umfange des benötigten Zahlengebietes sind $\triangle D$ - und $\eta$-Leiter nur kurz gehalten. An der $\eta$ Leiter ist auch die spezifische Zähigkeit ablesbar.

Die Benutzung der so entstandenen Tafel richtet sich nach dem Anwendungszweck. In der Regel wird man wie folgt verfahren:

Man sucht die gegebenen Werte für $\triangle D$ und $\eta$ auf, verbindet sie durch ein Lineal und findet so den zugehörigen F - Punkt. Nun legt man an diesen das Lineal an und kann dann wunschweise ablesen, welche Lebensdauer einer beliebigen gegebenen Teilchengröße entspricht, bei welcher Teilchengröße eine gegebene Dispersion haltbar wird u. s. f.

Die Tafel enthält durchaus nichts grundsätzlich Neues, sondern stellt nur einen Rechenbehelf dar, um die Rechenschwierigkeiten halber viel zu wenig benutzte Stokes'sche Formel allgemeinem Gebrauche zugänglich zu machen.

Eingezeichnet ist folgendes Beispiel:

Gegeben :

Unterschied der spezifischen Gewichte: $2,3-1,3=1,0, \triangle \mathrm{D}=1,0$

Innere Reibung: $\quad \eta=1,0$.

Gefunden: $[z \Leftrightarrow 90]$.

$$
[F \Leftrightarrow 0,005]
$$

Hiernach ist für :

$r$ oder $\mathrm{d} \quad \mathrm{v}$ bzw. $\mathrm{L}$ ca. ca.

$0,1 \mathrm{~cm} \quad 0,2 \mathrm{~cm} \quad 2 \mathrm{~m} / \mathrm{sek} . \quad, 5^{\prime \prime}$ $0,01 \mathrm{~cm} \quad 0,02 \mathrm{~cm} \quad 0,02 \mathrm{~m} / \mathrm{sek} .1 \mathrm{~min}$. $\begin{array}{llll}1,0 \mu & 2,0 \mu & 0,02 \mu / \text { sek. } & 14 \text { Tage }\end{array}$ $0,01 \mu \quad 0,02 \mu \quad 0,002 \mu \mu /$ sek. 5Jahrhunderte $1,0 \mu \mu \quad 2,0 \mu \mu$ sehr klein sehr groß.

\title{
Ueber schlauchförmige rhythmische Rosterscheinungen in einem Meteoreisen vom Cañon Diablo.
}

\author{
Von K. Endell (Berlin).
}

(Eingegangen am 27. Januar 1920.)

In seinem Buch "Geologische Diffusionen“ weist $R$. E. Lieseg ang ${ }^{1}$ ) verschiedentlich auf die Wichtigkeit der Annahme fester Diffusionsbahnen für die Entstehung "schalig-disperser Systeme " hin. Als ein charakteristisches Beispiel werden dort die Erzschläuche im Kalkstein bei Pitkäranta am Ladogasee erwähnt.

Durch Zufall fand ich der Form nach ähnliche Rostbildungen in einem Meteoreisen vom Cañon Diablo, das mir Herr Professor Joh. Königsberger als Studienobjekt für die optische Untersuchung von Metallegierungen nach seiner Methode ${ }^{2}$ ) liebenswürdiger Weise

1) R. E. Liesegang, Geologische Diffusionen (Dresden 1913) und Ueber schalig-disperse Systeme (Koll.-Zeitschr. 12, 74 und 269, 1913).

2) Joh. Königs berger, Centralbl. f. Min. 1908, 565,597 , und $1909,245$. überlassen hatte Als Beispiele von Diffusionsvorgängen in einem festen kristallisierten Medium scheinen sie mir einer kurzen Beschreibung wert.

Das Meteoreisen hatte einige Jahre in Watte verpackt gelegen, so daß das Rosten nur sehr langsam vorrücken konnte. Diesem Umstand ist es wohl auch zu verdanken, daß rhythmische Bildungen entstanden sind.

In Fig. 1 ist ein derartiges Gebilde in 20 facher Vergrößerung wiedergegeben. Fig. 2 zeigt das obere Ende in 40 facher Vergrößerung. Das Meteoreisen, das zur Erkennung anisotroper Bestandteile auf Hochglanz poliert war, hatte randlich raue Bruchflächen, von denen aus sich die Rostschläuche gewissermaken ins Eisen eingefressen haben. Sie sind von dunkelbrauner Farbe und lassen auf Grund der verschiedenen 
Reflexion ihre schalig-disperse Struktur gut erkennen.

$\mathrm{Dab}$ diese Rosterscheinungen nicht etwa durch bestimmte Gefügebestandteile des Meteor eisens in ihrer Richtung beeinfluBt waren, ergab die Untersuchung des geätzten Schliffs. Die sehr rasch vor sich gehende Aetzung geschah eine Minute lang in verdünnter, wässeriger Salpetersäure, wodurch freilich die rhythmischen Figuren teilweise zerstôrt wurden. Aus Fig. 3 ist deutlich $\mathrm{zu}$ ersehen, dab die Rostfíguren die körnig-flittrige Grundmasse des Meteoreisens völlig willkürlich durchsetzen. Diese besitzt Fetzenstruktur, die, wie die Untersuchungen von $F$ Berwerth ${ }^{3}$, sowie $F$. Rinne und $H$. E. Boeke ${ }^{4}$ ) zeigten, aus den für viele Meteoreisen charakteristischen Kamazitstreifen durch

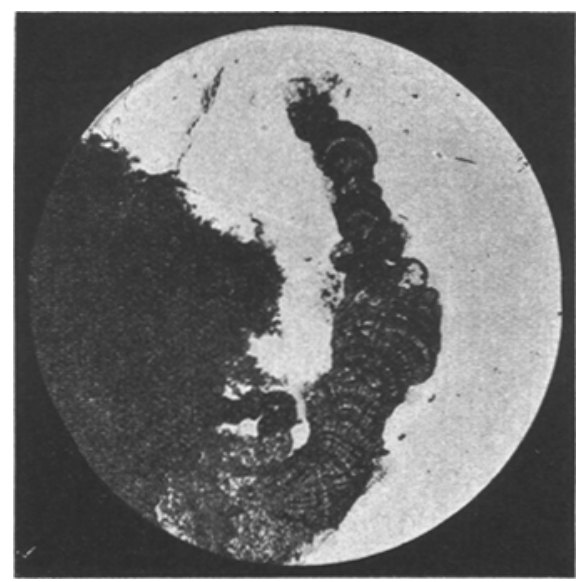

Fig. 1

Schlauchformige thythmische Rostfigur in poliertem Meteoreisen $(20 \times)$

Erhitzung entstehen kann. In dieser Eisennickellegierung wurden ferner Ausscheidungen, z. T. mit Eutektoidstruktur sowie Eisensulfideinschlüsse, beobachtet.

Das untersuchte Stück stammt vielleicht von dem Eisenkern der Eisenoxyd-Schalenkugeln, die G. P. Merril und W. Tas $\sin ^{5}$ ) vom Cañon Diablo beschreiben. Auch sie konstatierten darin das Fehlen breiter Kamazitstreifen und einen gröBeren Gehalt. an Eisenchlorid und Eisenphosphid, der die leichte Oxydierbarkeit bedingt, während das normale Eisen sehr widerstandsfähig ist. Die dort beschriebenen

3) F. B erwerth, S.B. Akad. Wiss. Wien 114 [I], 343 (1905).

4) F. Rinne und H. E. Boeke, N. Jahrb. Min. Festband 1907, 227.

5) G. P. Merill und W. Tassin, Smiths Misc. Coll. [4] 50, 203 (1907), ref. N. Jahrb. Min. 1909 [I], 354.
Schalenkugeln, die aus Brauneisen und Hämatit bestehen, entsprechen im großen vielleicht den mikroskopischen Rosterscheinungen.

Unter Zugrundelegung der elektrolytischen Rosttheorie des Eisens und der von R. E. Liesegang begründeten Theorie der Uebersättigung und Keimisolierung zur Erklärung der Rhythmogenie kann man sich die Rostfiguren folgendermaßen entstanden denken. Zunächst sei kurz der Rostvorgang als solcher im Sinne jener Theorie erläutert.

Die von $W$ ithne $y^{6}$ ) begründete, von $W$. H. Walker ${ }^{7}$ ), A. S. Cushman $n^{8}$ ) und andern weiter ausgebaute elektrolytische Theorie ist kurz folgende. Bedeckt sich Eisen mit einer Wasserhaut bzw. mit feuchter Luft, so entsteht, wie stets bei Berührung eines Metalls mit einer

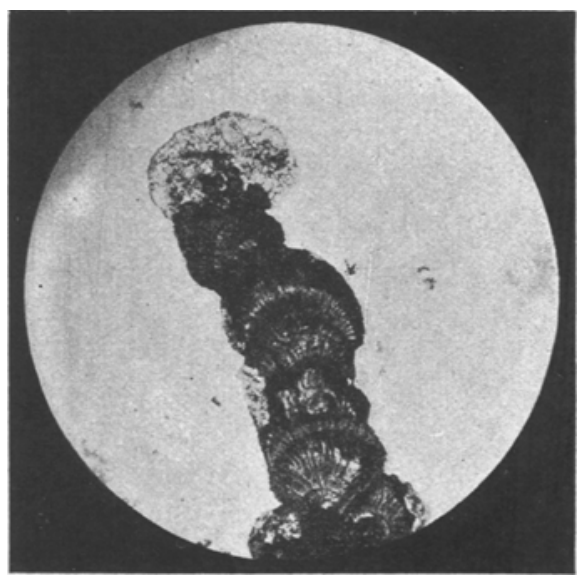

Fig. 2

Oberer Teil von Fig. $1(40 \times)$

leitenden Lösung, eine elektrische Spannungsdifferenz zwischen dem Metall und der Lösung Das Eisen gebt dabei in Form von Ferroionen in Lösung, während $\mathrm{H}$ - lonen aus dem schwach dissoziierten Wasser abgespalten werden. Die Kohlensäure der Luft wirkt wie jede andere Säure beschleunigend, da sie den Gehalt des Wassers an $\mathrm{H}$-lonen erhöht. Zwischen Eisen und Flüssigkeit findet dann ein Austausch elektrischer Ladungen statt. Wo die Eisenionen sich bilden, wird positive Elektrizität vom Eisen zur Lösung geführt und wo die H-lonen sich abscheiden und entladen, wird sie umgekehrt von der Lösung zum Eisen befördert.

6) Ch. Wit h ney, Journ. Amer. Chem. Soc. 25, 394 (1903)

7) W. H. Walke r, Journ. Amer. Chem. 29, 1251

(1907) uud Metallurgie 1909, 338.

8) A. S. C u s h m a n, Journ. Iron -Steel Inst. 1909, 33. 
Innerhalb des Metalls und der Flüssigkeit gleichen sich die Strömungen aus und es entsteht ein elektrischer Kreisstrom, der Lokalstrom. Spannungsunterschiede infolge von Verschiedenheiten der chemischen Zusammensetzung oder der Struktur der Oberfläche des Eisens begünstigen den Auflösungsvo gang. Damit aus dem elektrolytisch gelösten zweiwertigen Eisen der dreiwertige Rost, Eisenoxyd und Eisenoxydhydrat entsteht, muß es erst durch den in der feuchten Schicht enthaltenen Sauerstoff der Luft oxydiert werden. Der freiwerdende Wasserstoff wird wahrscheinlich zu Luft oxydiert. Ist erst einmal Rost gebildet, so dient dieser als Sauerstoffüberträger, der auch an seiner großen OberflächeO adsorbiert. Die Geschwindigkeit der. Rostbildungentspricht derAuflösungsgeschwindigkeit des Eisens, also der Stärke des Lokalstroms.

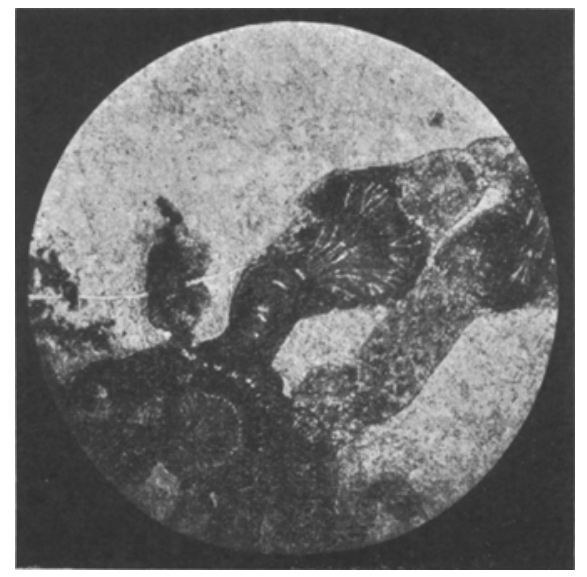

Fig. 3

Mit verd. Salpetersäure geätzter Schliff ( 40 fache Vergr.). Die Rostfiguren durchsetzen willkürlich die körnigflittrige Grundmasse.

Im vorliegenden Meteoreisen begünstigen die vorhandenen Beimengungen, wie Sulfíde, Phosphide usw. die Rostbildung. Bezüglich dieser Beimengungen ist das Eisen, wie J.W. $\mathrm{Cobb}^{9}$ ) experimentell nachwies, elektrisch positiv und geht in Lösung, während die Beimengungen ungelöst bleiben. Durch chemische und mikroskopische Beobachtungen konnte der Nachweis erbracht werden, dab Eisen beim Kontakt mit diesen Stoffen, zu denen auch der neugebildete Rost zu rechnen wäre, in Gegenwart einer

9) J. W. Cob b, Journ. Iron-Steel Inst. 1911, 170. In Fig. 9 dieser Abhandlung sind rhythmische Fällungen abgebildet, die von Eisenphosphid ausgehend auf der Eisenoberfläche entstanden sind. elektrisch leitenden Flüssigkeit in Lösung geht. Und zwar ist dieser Auflösungsvorgang mehr von der Qualität und Verteilung der Beimengungen als von ihrer Quant ität a bhängig. So widersteht ein möglichst homogenes, wenn auch chemisch ziemlich unreines, Eisen der Korrosion viel besser, als ein chemisch reineres Material mit stark inhomogener Struktur. Nach diesen Beobachtungen Cobb's ist es klar, daß die Fetzenstruktur des Meteoreisens infolge der vielen Zentren und der dadurch bedingten (beim Kontakt mit Eisensulfid und -phosphid besonders starken) Lokalströme den Rostvorgang beschleunigen.

Der Rhythmus der Rostfiguren läßt sich durch Uebersättigung und Keimisolierung erklären. Als Ausgangspunkt diente bereits vorhandener Rost am rauhen, nicht polierten Rand des Stückes. Durch Lokalstrombildung geht Eisen in Form von Ferroionen in Lösung. Das durch Luftzutritt oxydierte Eisen bleibt bis zur Erreichung der metastabilen Grenze gelöst, fällt nach deren Ueberschreitung als $\mathrm{Fe}(\mathrm{OH})_{3}$ aus und lagert sich an Ort und Stelle als Rost ab. Infolge der Starrheit des Mediums, in dem sich der Vorgang abspielt, und des geringen Lösungsmittels, werden die Rostkeime isoliert, so daB von neuem Uebersättigung eintreten kann. Die zunächst gebildete Rostschicht bedingt weitere Auflösung von Eisen, das sich in gewisser Entfernung annähernd konzentrisch ablagert u. s. f. Der dazwischen liegende Raum wird wohl erst nachträglich von strahligem Eisenoxyd ausgefüllt. Auf jeden Fall ist der Rost in den rhythmischen Zonen sehr viel dichter als in den Zwischenräumen. Die schlauchförmige Gestalt ist wahrscheinlich dadurch ermöglicht worden, daß̣ die Auflösungsgeschwindigkeit des Eisens, also auch die Rostbildungsgeschwindigkeit beim Kontakt des Eisens miț bereits vorhandenem Rost gröBer ist als mit Beimengungen. Wenigstens gingen sämtliche derartigen Gebilde von bereits randlich vorhandenem Rost aus.

Sogenannte Jahresringe in einem Bleirohr, das 24 Jahre $1 \mathrm{~m}$ tief in der Erde gelegen hatte, die E. J. Kohlmey e ${ }^{10}$ ), beschrieben hatte, bringt $R$. E. Li es egang gleichfalls mit rhythmischen Fällungen und Diffusionsvorgängen in Zusammenbang. Wahrscheinlich spielt ein intermediârer elektrôlytischer Lösungsvorgang auch in diesem Fall eine Rolle.

Berlin 1914.

Eisenhüttenmännisches Laboratorium der Technischen Hochschule.

10) J. Koh 1 meyer, Chem.-Ztg. 1912, 993. 